\title{
Optical response of two-dimensional few-electron concentric double quantum rings: A local-spin-density-functional theory study
}

\author{
F. Malet, M. Pi, M. Barranco, and E. Lipparini* \\ Departament ECM, Facultat de Física, and $I N^{2}$ UB, Universitat de Barcelona, Diagonal 647, 08028 Barcelona, Spain \\ Ll. Serra \\ Departament de Física, Universitat de les Illes Balears, and Institut Mediterrani d'Estudis Avançats IMEDEA (CSIC-UIB), \\ E-07122 Palma de Mallorca, Spain
}

(Received 24 August 2006; revised manuscript received 23 October 2006; published 16 November 2006)

\begin{abstract}
We have investigated the dipole charge- and spin-density response of few-electron two-dimensional concentric nanorings as a function of the intensity of a perpendicularly applied magnetic field. We show that the dipole response displays signatures associated with the localization of electron states in the inner and outer ring favored by the perpendicularly applied magnetic field. Electron localization produces a more fragmented spectrum due to the appearance of additional edge excitations in the inner and outer ring.
\end{abstract}

DOI: 10.1103/PhysRevB.74.193309

PACS number(s): 73.21.-b, 73.22.-f, 71.15.Mb

Progress in nanofabrication technology has allowed one to produce self-assembled, strain-free, nanometer-sized quantum complexes consisting of two concentric, welldefined GaAs/AlGaAs rings ${ }^{1,2}$ whose theoretical study has recently attracted some interest. ${ }^{3-6}$ Most of these works are concerned with the properties of their ground state, although the optical properties of one- and two-electron concentric double quantum rings (CDQR) at zero magnetic field have been addressed using a single-band effective-mass envelope model discarding Coulomb correlation effects. ${ }^{2}$

The singular geometry of CDQR has been found to introduce characteristic features in the addition spectrum compared to that of other coupled nanoscopic quantum structures. As a function of the interring distance, the localization of the electrons in either ring follows from the interplay between confining, Coulomb, and centrifugal energies. Each of them prevail in a different range of interring distances, affecting in a different way the CDQR addition spectrum. ${ }^{6}$ It is thus quite natural to investigate whether and how localization effects may show up in the dipole response, using a perpendicularly applied magnetic field instead of the interring distance to control the electron localization in either ring.

The aim of this paper is to use local-spin-densityfunctional theory (LSDFT) as described in detail in Ref. 7, to investigate the dipole longitudinal response of CDQR. The method has been used in the past to address the response of single quantum rings (see, e.g., Ref. 8 and references therein). We address here the few-electron case, and consider the CDQR's as strictly two-dimensional systems.

Within LSDFT, the ground state of the system is obtained by solving the Kohn-Sham (KS) equations. The problem is simplified by the imposed circular symmetry around the $z$ axis, which allows one to write the single particle (sp) wave functions as $\varphi_{n l \sigma}(\mathbf{r}, \sigma)=u_{n l \sigma}(r) e^{-l l \theta} \chi_{\sigma}$, being $-l$ the projection of the sp orbital angular momentum on the $z$ axis. The confining potential has been taken in a form that slightly generalizes that of Ref. 4,

$$
V_{c f}(r)=\min \left\{\frac{1}{2} m \omega_{1}^{2}\left(r-R_{1}\right)^{2}, \frac{1}{2} m \omega_{2}^{2}\left(r-R_{2}\right)^{2}\right\},
$$

with $R_{1}=20 \mathrm{~nm}, R_{2}=40 \mathrm{~nm}, \omega_{1}=30 \mathrm{meV}$, and $\omega_{2}=40 \mathrm{meV}$. The radii have been fixed to the experimental values, ${ }^{2}$ while the frequencies are rather arbitrary. We have considered large frequencies to mimic the strong confinement felt by the CDQR, and have taken $\omega_{2}>\omega_{1}$ to somewhat compensate that, as $R_{2}>>R_{1}$, the "surface" of the outer ring might have been overestimated if we had taken both frequencies equal. In three dimensions, more realistic confining potentials, better suited to model the experimental devices, ${ }^{2}$ have been considered. ${ }^{6}$ On the CDQR system it may act a constant magnetic field $B$ in the $z$ direction, to which a cyclotron frequency $\omega_{c}=e B / m c$ is associated. We have taken for the dielectric constant, electron effective mass, and effective gyromagnetic factor, the values appropriate for GaAs, namely, $\epsilon=12.4, m^{*}=0.067$, and $g^{*}=-0.44$, and have solved the KS equations for up to $N=6$ electrons, and for $B$ values up to 4-5 T, depending on $N$. In the following, we discuss some illustrative results.

Figure 1 shows the squared wave functions $|u(r)|^{2}$ corresponding to the KS occupied orbitals for $N=6$ and $B=0 \mathrm{~T}$ (top panel). In this case, the total spin of the CDQR is zero, and the spin-up and -down orbitals corresponding to the same $(n,|l|)$ values are degenerate. As a consequence, there are only two different radial wave functions, one for $s(l=0)$ states, and another for $p(l= \pm 1)$ states. It can be seen that electrons are fairly delocalized within the CDQR; for the chosen confining potential, localized configurations would only appear at larger interring distances. ${ }^{6}$

It is known that for an $N$-electron single quantum ring, sp states with small $l$ values become progressively empty as $B$ increases. This can be intuitively understood as follows. If only nodeless radial states are occupied, the Fock-Darwin wave functions are proportional to $x^{|l|} e^{-x^{2} / 4}$, where $x=r / a$, being $a=\sqrt{\hbar /(2 m \Omega)}$ with $\Omega=\sqrt{\omega_{0}^{2}+\omega_{c}^{2} / 4}$. Of course, this is so for a harmonic confining potential of frequency $\omega_{0}$, and not for the ring confining potential, but some of that wave function structure remains even in this case. These wave 


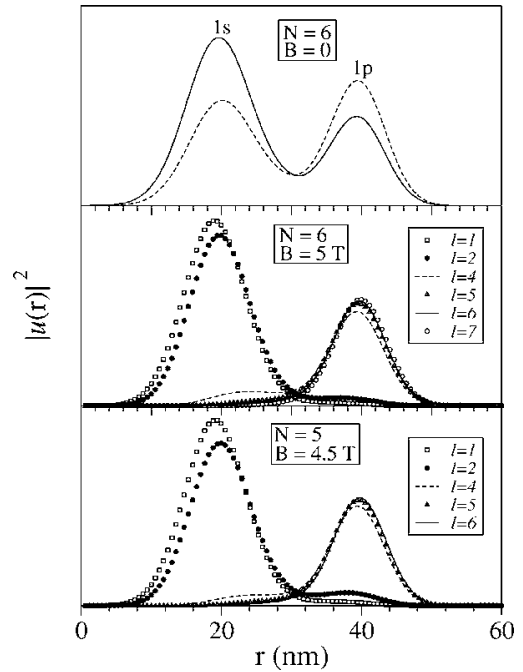

FIG. 1. Top panel: Squared wave functions (arbitrary units) as a function of $r(\mathrm{~nm})$ of the KS occupied orbitals corresponding to $N=6, B=0 \mathrm{~T}$. Middle panel: Same as top panel for $N=6, B=5 \mathrm{~T}$; all orbitals are spin polarized. Notice that the $l=3$ orbital is not occupied. Bottom panel: Same as middle panel for $N=5, B=4.5 \mathrm{~T}$

functions are peaked at $r_{\max } \sim \sqrt{2|l|} a$, and consequently, as $B$ increases, the $|l|$ values corresponding to occupied levels must increase so that $r_{\max }$ sensibly lies within the range of $r$ values spanned by the ring morphology. The same appears to happen for the CDQR we have studied, as illustrated in Fig. 1. The squared wave functions $|u(r)|^{2}$ for $N=6, B=5 \mathrm{~T}$, and for $N=5, B=4.5 \mathrm{~T}$ are displayed in the middle and bottom panels of this figure, respectively, showing that indeed, high$l$ orbitals are mostly localized in the outer ring, whereas low$l$ orbitals are mostly localized in the inner ring. A complete localization of all orbitals could have been achieved using, e.g., a larger $\omega_{1}$ value.

We show in Fig. 2 the sp energy levels as a function of $l$ for $N=5$ and several $B$ values. Upward (downward) triangles correspond to spin-up (down) orbitals. The sp energies are distributed into paraboliclike bands, each one corresponding to a different value of the radial quantum number $n$. It can be seen that in most cases, spin-up and -down orbitals corresponding to the same values of $(n, l)$ are not degenerate due to the spin magnetization dependence of the exchangecorrelation energy - we recall that $N$ is odd. Yet, some orbitals still present this degeneracy, and among them, some are occupied orbitals, such as the $[(0,1) \uparrow, \downarrow]$ ones at $B=1 \mathrm{~T}$, or the $[(0,2) \uparrow, \downarrow]$ ones at $B=2 \mathrm{~T}$. This can be explained from the different spatial localization of these orbitals in the $\mathrm{CDQR}$ and the distribution of the spin magnetization $m(r)$ $=n^{\uparrow}(r)-n^{\downarrow}(r)$, as shown in Fig. 3: at $B=2 \mathrm{~T}$, the $l= \pm 2 \mathrm{sp}$ orbitals are mostly localized in the outer ring, in which the local magnetization is fairly small, whereas at $B=3 \mathrm{~T}$, the $l$ $= \pm 1 \mathrm{sp}$ orbitals are mostly localized in the inner ring, in which the local magnetization is fairly small. Eventually, the number of spin-up orbitals is so large than the $\uparrow, \downarrow$ degeneracy is fully broken (this happens for $B>3$ in the displayed cases).

Electron localization in the inner or outer ring induced by the magnetic field thus shows up in the KS orbitals, in spite

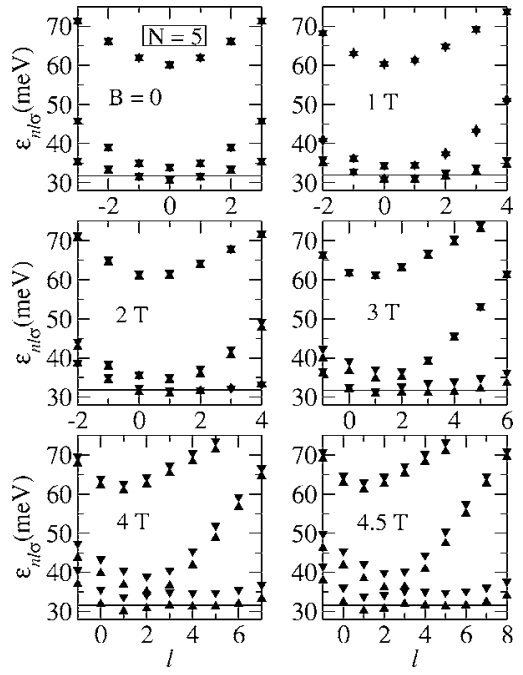

FIG. 2. Kohn-Sham sp energies (meV) of the $N=5 \mathrm{CDQR}$ ground state as a function of $l$ for several $B$ values. Upward (downward) triangles correspond to spin-up (down) orbitals. The thin horizontal line indicates the Fermi level.

of having solved the KS equations implicitly assuming a full coherence regime in which electrons are allowed to occupy the whole $\mathrm{CDQR}$ surface. Yet, they can be localized in either ring if the resulting configuration has a lower energy. It is relevant for the analysis of the dipole response to notice that electron localization is best achieved when, due to the double well structure of the confining CDQR potential, KS orbitals corresponding to an intermediate $l$ value are not occupied. In the present case, it happens for $l=3$. Intuitively, the missing $l$ is the one whose KS orbit has a "radius" similar to that of the maximum of the interring barrier.

A full delocalization situation produces a very regular $\mathrm{sp}$ energy pattern since, due to it, electrons "feel" simultaneously the confining potential produced by both constituent rings. This happens, e.g., for $B=0$ and other low- $B$ values. The situation may change at higher magnetic fields. Indeed, Fig. 2 shows that around $B=3 \mathrm{~T}$, the two lowest paraboliclike bands tend to cross between $l=2$ and 3 . Roughly speaking, each paraboliclike band arises from one of the constituent rings. The crossing is quantum mechanically prevented-

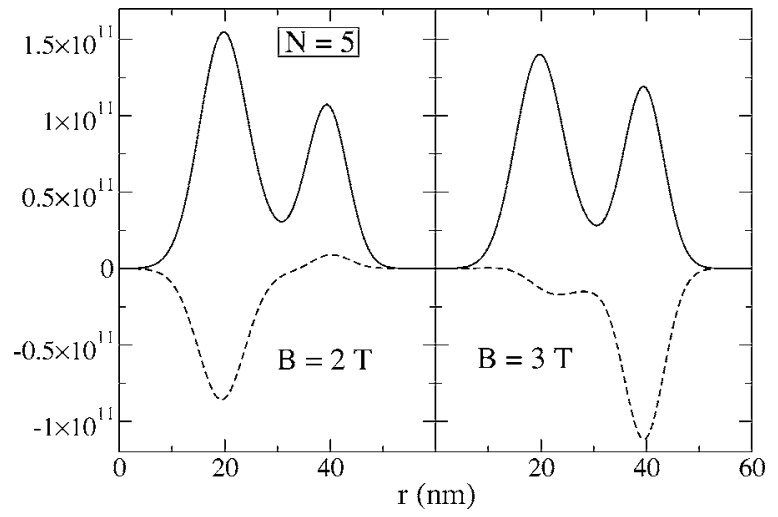

FIG. 3. Electron density $n(r)$ (solid line) and spin magnetization $m(r)$ (dashed line) in $\mathrm{cm}^{-2}$ for the $N=5 \mathrm{CDQR}$. 


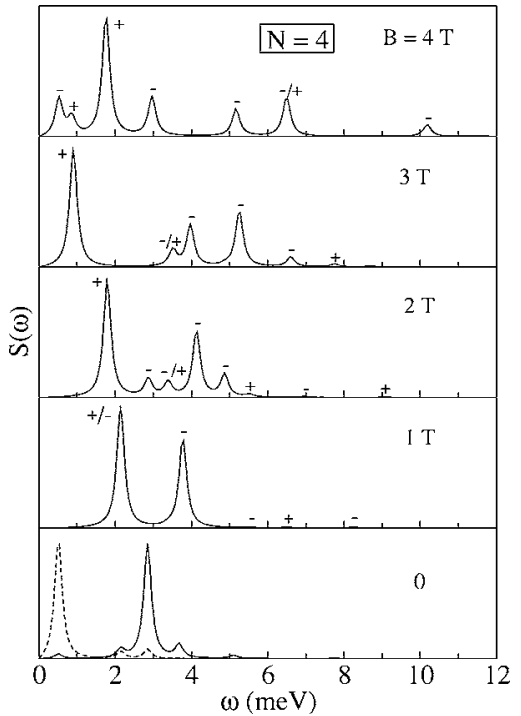

FIG. 4. Charge dipole strength (solid lines, arbitrary units) and spin dipole strength (dashed lines, arbitrary units) for the $N=4$ CDQR as a function of the excitation energy $(\mathrm{meV})$ and several $B$ values. Some structures are superposition of peaks of different polarization, which is represented by a " " symbol with the polarization of the more intense peak indicated to the left of the slash. The intensities are fixed in such a way that for a given $B$, the more intense peaks in both channels roughly have the same height.

level repulsion. The same happens at $B=4$ and $4.5 \mathrm{~T}$. A similar effect was found in Ref. 6, but described in terms of the interring distance instead of $B$. Band crossing prevented by level repulsion also appears for the one-electron CDQR at similar magnetic fields. In this case, spin-up and -down orbitals are nearly degenerate due to the smallness of the Zeeman energy.

Once the ground state has been determined, the dipole response can be worked. It is experimentally known ${ }^{9,10}$ that, for one single quantum ring, the dipole spectrum as a function of $B$ consists of several high- and low-frequency branches. For large $B$, the low-frequency branches are identified as edge magnetoplasmons localized at the inner and outer ring boundaries.

In the delocalized regime, the physical pictures for a single quantum ring and for a $\mathrm{CDQR}$ turn out to be similar. The magnetoexcitations can be classified into bulk (highenergy) and edge (low-energy) modes, with delocalization producing only two effective edges: the inner edge of the smaller ring and the outer edge of the larger ring. The origin of the high- and low-frequency peaks can be easily understood from, e.g., the $B=1$ and $2 \mathrm{~T}$ panels in Fig. 2. Bulk, high energy peaks arise from non-spin-flip electronic excitations mostly involving $\Delta n=1, \Delta l= \pm 1$ changes (inter "Landau level" excitations), while edge, low-energy peaks arise from non-spin-flip electronic excitations involving $\Delta n=0$, $\Delta l= \pm 1$ changes (intra "Landau level" excitations). One thus would expect that the $B$ dispersion of bulk and edge modes split into two branches each, one corresponding to $\Delta l=+1$ [circularly polarized $(+)$ excitations], and another to $\Delta l=$ -1 [circularly polarized (-) excitations]. The (-) edge modes are intra Landau level excitations of the innermost

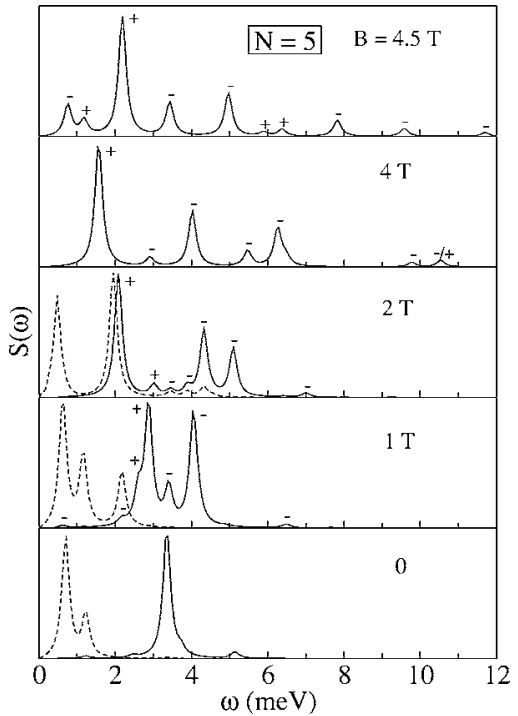

FIG. 5. Same as Fig. 4 for $N=5$.

boundary of the double ring system, whereas the $(+)$ edge modes are intra Landau level excitations of the outermost boundary. The high-energy modes are bulk modes mostly of (-) character, similarly to the cyclotron mode in quantum dots and wells, and carry much less strength, i.e., are less intense. Notice that at $B=0$ the $( \pm)$ excitations are degenerate.

Examples of this behavior as a function of $B$ are shown in Figs. 4-6 for $N=4,5$, and 6, respectively. The solid lines in these figures represent the charge dipole strength $S(\omega)$ in arbitrary units as a function of the excitation energy. At low $B$ values, the presence of two edge modes with opposite circular polarizations can be seen, together with fairly weak structures arising from $\Delta n=1$ transitions. The figures only represent the low energy (up to $12 \mathrm{meV}$ ) part of the dipole spectrum. Some strength is also in the form of low intensity, higher energy modes arising from $\Delta n=2$ electronic excitations, not shown in these figures, easily identifiable in plots

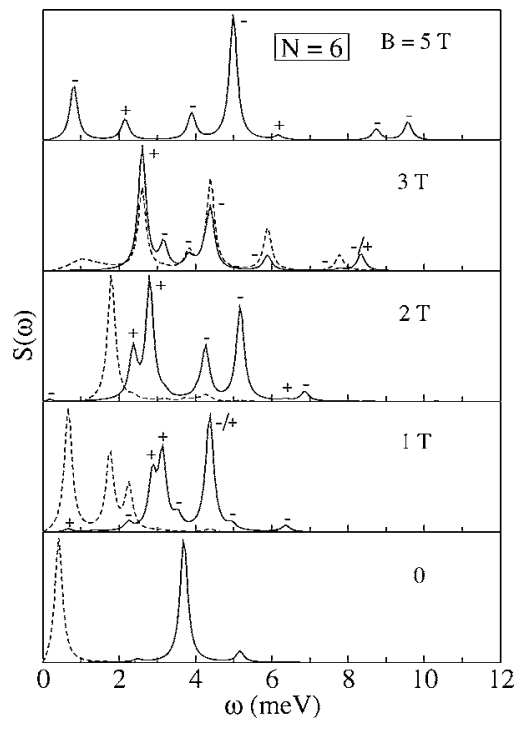

FIG. 6. Same as Fig. 4 for $N=6$. 
as those shown in Fig. 2. Some modes present a fine structure (fragmentation), despite the tendency of the electronhole interaction to correlate the free-electron excitations, grouping them coherently into few excitation peaks.

The spin dipole strength is also represented in these figures in arbitrary units (dashed lines). Obviously, when the CDQR is fully polarized both strengths coincide and we have plotted only one of them. As a general rule, charge modes are at higher energies than spin modes because the electron-hole interaction is repulsive in the density channel, as it is essentially determined by the electron-electron Coulomb interaction, whereas it is attractive in the spin channel, as it is determined by the attractive exchange-correlation interaction. It can be seen from Figs. 4-6 that in some situations, spin and charge strengths are coupled. This coupling may appear when the ground-state configuration has a nonzero total spin. $^{7}$

Finally, we discuss the case of localized electrons, exemplified here by the $N=4, B=4 \mathrm{~T}$, the $N=5, B=4.5 \mathrm{~T}$, and the $N=6, B=5$ T CDQR's. In this regime the response of the system deviates from that of the single ring. The fact that the $l=3 \mathrm{KS}$ orbital is empty makes it possible to generate additional low-energy modes at the inner and outer boundaries of both rings. One thus would expect the appearance of a richer dipole strength, with two series of $(-)$ and $(+)$ polarization edge modes instead of just one. This is indeed what is shown in the corresponding panels of Figs. 4-6 and it signals the onset of electron localization in the strength function.

The present study can be extended to the case of manyelectron CDQR and to other multipole excitations, or to incorporate on-plane wave-vector effects for the analysis of Raman experiments. ${ }^{8}$ A more realistic description of the CDQR confining potential ${ }^{6}$ demands a three-dimensional approach. ${ }^{11}$ Work along this line is in progress.

We would like to thank Josep Planelles for useful discussions. This work has been performed under Grants No. FIS2005-01414 and No. FIS2005-02796 from DGI (Spain) and No. 2005SGR00343 from Generalitat de Catalunya. E.L. has been suported by DGU (Spain), Grant No. SAB20040091.
*Permanent address: Dipartimento di Fisica, Università di Trento, and INFN, 38050 Povo, Trento, Italy.

${ }^{1}$ T. Mano, T. Kuroda, S. Sanguinetti, T. Ochiai, T. Tateno, J. Kim, T. Noda, M. Kawabe, K. Sakoda, G. Kido, and N. Koguchi, Nano Lett. 5, 425 (2005).

${ }^{2}$ T. Kuroda, T. Mano, T. Ochiai, S. Sanguinetti, K. Sakoda, G. Kido, and N. Koguchi, Phys. Rev. B 72, 205301 (2005).

${ }^{3}$ G. Fuster, M. Pacheco, and Z. Barticevic, Braz. J. Phys. 34, 666 (2004).

${ }^{4}$ B. Szafran and F. M. Peeters, Phys. Rev. B 72, 155316 (2005).

${ }^{5}$ J. Planelles and J. I. Climente, Eur. Phys. J. B 48, 65 (2005).

${ }^{6}$ J. I. Climente, J. Planelles, M. Barranco, F. Malet, and M. Pi,
Phys. Rev. B 73, 235327 (2006).

${ }^{7}$ Ll. Serra, M. Barranco, A. Emperador, M. Pi, and E. Lipparini, Phys. Rev. B 59, 15290 (1999).

${ }^{8}$ A. Emperador, M. Pi, M. Barranco, and E. Lipparini, Phys. Rev. B 64, 155304 (2001).

${ }^{9}$ C. Dahl, J. P. Kotthaus, H. Nickel, and W. Schlapp, Phys. Rev. B 48, 15480 (1993).

${ }^{10}$ A. Lorke, R. J. Luyken, A. O. Govorov, J. P. Kotthaus, J. M. Garcia, and P. M. Petroff, Phys. Rev. Lett. 84, 2223 (2000).

${ }^{11}$ M. Pi, F. Ancilotto, E. Lipparini, and R. Mayol, Physica E (Amsterdam) 24, 297 (2004). 